\title{
Japon. Découverte de manuscrits bouddhiques chinois au Japon
} Conférence prononcée par Monsieur Ochiai Toshinori

Frédéric Girard, Liying Kuo

\section{Citer ce document / Cite this document :}

Girard Frédéric, Kuo Liying. Japon. Découverte de manuscrits bouddhiques chinois au Japon. In: Bulletin de l'Ecole française d'Extrême-Orient. Tome 83, 1996. pp. 368-375;

doi : https://doi.org/10.3406/befeo.1996.3809

https://www.persee.fr/doc/befeo_0336-1519_1996_num_83_1_3809

Fichier pdf généré le 08/02/2019 
pour les études coréennes en France, de maintenir et de développer les activités du centre EFEO de Séoul dans le cadre d'une coopération scientifique accrue avec les chercheurs coréens, d'animer des programmes collectifs de recherche avec les collègues coréens, portant tout aussi bien sur la culture coréenne proprement dite que sur les études chinoises, japonaises ou sud-est asiatiques.

Pierre F. SOUYRI

\section{JAPON}

\section{Découverte de manuscrits bouddhiques chinois au Japon Conférence prononcée par Monsieur Ochiai Toshinori}

Monsieur Ochiai Toshinori, professeur au Kajō Junior College de Kyōto (Japon), dirige une série où sont publiés des manuscrits bouddhiques copiés à la fin de l'époque Heian (XIe $X I I{ }^{e}$ siècle), conservés dans un monastère bouddhique à Nagoya, les Nanatsu-dera'. Ces manuscrits sont pour la plupart des sūtra rédigés en Chine entre IVe et VII siècle et jamais imprimés. Certains sont des auvres inconnues à ce jour. Leur découverte est due à M. Ochiai. À la demande de l'URA 1063 du CNRS, et de l'EFEO, M. Ochiai a obtenu une bourse du Ministère l'Enseignement Supérieur et de la Recherche pour un séjour de deux mois à Paris. Durant son séjour (15 août - 14 octobre 1995) il a travaillé avec les chercheurs du programme d'étude des textes apocryphes de l'URA 1063 et de l'EFEO. On trouvera cidessous le texte de sa conférence donnée pour le grand public aux Instituts d'Extrême-Orient du Collège de France, le 28 septembre 1995. Sa conférence fut prononcée en japonais; elle a été traduite par M. Frédéric Girard et Kuo Li-ying. Ce texte constitue une introduction aux collections monastiques japonaises de copies de textes bouddhiques; la plupart ne sont pas encore bien répertoriées. $M$. Ochiai a attiré l'attention sur ces collections qui conservent des versions plus anciennes que celles utilisées pour éditer le canon dit Taishō. Il a essayé d'expliquer pourquoi les originaux de ces textes n'existent plus en Chine. Ses arguments nous paraissent parfois discutables. Ils méritent toutefois d'être examinés.

1. Ochiai Toshinori, Manuscrits of Nanatsu-dera. A Recently Discovered Treasure-House in Downtown Nagoya, with related remarks by Makita Tairyō and Antonino Forte, translated and edited by Silvio Vita, Kyōto : Italian School of East Asian Studies, Occasional Papers 3, 1991, 102 p. + 18 fig. ; Makita Tairyō 钽田卸. 諦亮 et Ochiai Toshinori 落合俊典 (éd.), Nanatsu-dera kōitsu kyōten kenkyū sōsho 七寺古逸經典研究裳書 (The Long Hidden Scriptures of Nanatsu-dera, Research Series) I : Chūgoku senjutsu kyōten (sono ichi) 中國撰述經其 (其之一) (Scriptures Composed in China, volume I), Tōkyō: Daitō 大東, 1994, 536 p.; idem, vol. III, 1995, 1278 p. Un compte-rendu des deux premiers livres a été fait par Kuo Li-ying, BEFEO, vol. 82 (1995), p. 38-44. 


\section{Texte de la conférence}

\section{La transmission du canon bouddhique de Chine au Japon}

Il est difficile de savoir exactement à partir de quand et dans quelles circonstances les sūtra bouddhiques chinois ont été transmis au Japon pour la première fois ${ }^{1}$. On a une connaissance plus précise de la diffusion des textes bouddhiques à partir de l'époque de Nara (710-784). De nombreux matériaux provenant du «Bureau de copie des sütra bouddhiques » (富經所) sont en effet conservés dans le célèbre Shōsōin 正倉院, trésor de la famille impériale ${ }^{2}$. Les recherches faites jusqu'ici n'ont concerné que les documents qui en proviennent.

En 735, le moine japonais Genbō玄昉 [?-746] a apporté de la capitale chinoise, Chang'an, une version complète du canon (一切經), en 5048 rouleaux, conforme au Catalogue de l'ere Kaiyuan (Kaiyuan Shijiao lu開元䆁教錄) compilé en 730 par le moine chinois Zhisheng 智昇 [669-740] [T. 2154]. Ce canon semble être une copie de la version effectuée à la cour impériale de Chang'an. D'autres copies du canon bouddhique ont existé antérieurement au Japon. Elles correspondaient au Catalogue des livres bouddhiques des Grands Tang (Da Tang neidian lu 大唐內典錄) [T. 2149], [compilé en 664] par Daoxuan 道宣 [596-667], mais les textes utilisés pour ce canon sont de provenances diverses et, par voie de conséquence, très divers. En effet ces textes ont été peu à peu apportés au Japon grâce aux efforts incessants de moines érudits japonais : certains provenaient de la péninsule coréenne, d'autres de la Chine, mais ils différaient des versions recopiées sous l'égide de la cour chinoise. De nombreuses questions de philologie subsistent les concernant.

En possession d'un canon constitué en bonne et due forme, la cour impériale japonaise a patronné une entreprise massive de copie de ce canon. C'est l'origine de la célèbre copie du canon bouddhique exécutée à l'instigation de l'impératrice Kōmyō光明皇忈 [701-760] à partir de 740. Les exemplaires copiés à Chang'an par la cour chinoise sont perdus, mais nombre de textes qui en ont été recopiés d'après les copies chinoises au Japon par des scribes officiels et des moines nous sont heureusement parvenus.

De nouvelles traductions [et rédactions] d'ouvrages bouddhiques sont continûment apparues en Chine. Des moines japonais se sont efforcés de les rapporter au Japon. Tel est le cas de religieux comme Saichō最澄 (767-822) et Kūkai 空海 (774-835), qui ont ainsi transmis très tôt beaucoup de textes jusqu'alors inconnus sur le sol japonais.

\section{Histoire de la copie des textes bouddhiques au Japon}

Au Japon, la plus ancienne copie de texte aujourd'hui connue date de 686. C'est le Recueil de dhārani de l'aire de diamant (Jingangchang tuoluoni jing金, 湖場陀纙尼經) [T. 1345]. Nombre d'autres textes ont dû être copiés antérieurement de façon irrégulière. À partir de l'époque de Nara des copies du canon ont été entreprises systématiquement et régulièrement sous l'autorité de l'État. Jusqu'à présent, on n'a guère prêté attention aux copies de textes mentionnés dans la « liste des ouvrages non inclus dans le canon » [T. 2157 (30), LV, 1046b1048a : 不入藏目錄]. Ces textes étaient considérés comme faisant double emploi dans les catalogues bouddhiques chinois [infra], et donc au Japon où on suivait le modèle continental.

1. Les sources anciennes font état de deux dates pour l'introduction du bouddhisme, à titre officiel : 552 (Nihon shoki 日本書紀, “Annales du Japon») et 538 ( Biographie du Prince Shōtoku 》 上宮聖德法王帝説 et 《Historique et catalogue des biens du Gangōji » 元興寺伽藍緣起并流記資材帳). On préfère en général cette dernière date.

2. Shōsōin monjo正倉院文書, «Archives du Shōsōin », in Dainihon komonjo大日本古文書, « Archives anciennes du Grand Japon», 1-25. Cf. Ishida Mosaku 石国茂焦, Shakyō yori mitaru Nara bukkyō no kenkyū 寫經より兄たる奈良佛教の研究 (“Recherches sur le bouddhisme de Nara d'après les copies des textes bouddhiques »), Tökyō : Tōyōbunko, 1930 (réimpression 1966). 
Cependant, il existait un bureau d'examen des textes, où l'on collationnait ces textes écartés du canon.

Le travail de copie du canon a connu au Japon un regain de succès aux XI ${ }^{\mathrm{e}}$ et XII ${ }^{\mathrm{e}}$ siècles : environ quarante de ces entreprises sont attestées; elles s'appuient sur le Catalogue de l'ère Zhenyuan (Zhenyuan lu 真元錄) compilé en 800 [T. 2157] pour la plus grande partie, mais utilisent également des textes de l'édition compilée sur ordre impérial sous les Song du Nord entre 971 et 983 . Celle-ci se fonde sur le Catalogue de l'ère Kaiyuan, compilé en 730. Cependant, on a peu recopié de textes non intégés dans le canon.

Seuls subsistent quelques exemplaires. En voici un aperçu, monastère par monastère :

1. Le Tōji 東寺. 52 titres en 137 rouleaux d'ouvrages répertorićs comme « exclus du canon 》 (不入藏經) dans le Catalogue du canon du Tōji 東寺一切經目錄) [Taishō : Shōwa hōbō sōmokuroku昭和法總目錄, vol. I, 1015b-1016a]. Or, le catalogue chinois de l'ère Zhenyuan donne 118 titres en 247 rouleaux de textes exclus du canon [T. 2157 (30), LV, $1046 \mathrm{~b}-1048 \mathrm{a}$ ]. Il manque donc 66 titres et 110 rouleaux. Il est intéressant de noter que le Catalogue du Tōji mentionne 16 titres en 60 rouleaux de textes rares qui ne correspondent pas au catalogue des Tang [idem, vol. I, 1016a-c : 露錄不合經 $]^{1}$.

2. Les Nanatsudera 七寺 à Nagoya. 13 titres en 105 rouleaux de textes écartés du canon. Le canon des Nanatsudera s'appuie sur la copie conservée au Hōshōji法勝寺 du Catalogue de l'ère Zhenyuan. On peut le déduire du fait qu'il porte dans les colophons écrits sur l'exemplaire du même catalogue conservé aux Nanatsudera, au-dessus des titres recopiés, la mention : version du Hōshōji, cochée avec un trait rouge à droite ; version de Fushimi (inconnue), cochée avec un trait rouge à gauche ; version du Bonshakuji, cochée avec un rond noir au centre (右點 法腼寺 金字經.左點 伏見本.星點 焚釋寺本).

Le canon du Hōshōji est perdu. Le Hōshōji était un temple fondé sur le vœu de l'empereur Shirakawa (1053-1129), qui héritait de la tradition des grands temples de Nara (Tōdaiji 東大寺 et Kōfukuji 舅福寺).

3. Le Saihōji 西方寺. 2629 rouleaux au total. Sa copie du canon (dite aussi du Daimonji 大門寺一切經) se fonde sur 《la liste de textes inclus dans le canon (入藏錄) 》 du Catalogue de l'ère Zhenyuan [T. $2157(29,30), \mathrm{LV}, 1024 \mathrm{a}-1056 \mathrm{a}]$, en ajoutant 6 titres en 6 rouleaux de plus. Le canon de Saihōji renferme un copie de la Biographie du bodhisattva Aśvaghoșa (Maming pusa zhuan 馬鸣菩薩㴬) [T. 2046] et une autre du 9e et $10^{\mathrm{e}}$ juan du Grand sütra des noms de buddha (Da forning jing大佛名經) [cf. T. 441]. À partir de ces deux copies, on peut déduire la provenance de l'original : les copies du Saihōji appartiennent à la tradition de Nara et sont fondées sur le choix du Catalogue de l'ère Zhenyuan.

4. Le Kōshōji 興型寺 [temple de la secte Rinzai 臨济 de Kyōto]. 4000 rouleaux copiés pour la plupart après l'époque de Heian selon les recherches faites par Oya Tokujō大屋德城. Y figurent également des copies manuscrites et des livres imprimés des époques Kamakura (1183-1331) et Muromachi (1392-1569). Une copie (colophon daté de 785) du premier rouleau de la Relation sur les contrées occidentales (Ta Tang Xiyu ji 大唐西域記) [T. 2087] a été exécutée à l'époque de Nara. Toutes ces copies se présentent sous forme de livres à soufflet et ont été rangées dans des coffrets en bois. Cependant, l'ordre en a été perturbé et le classement a été refait par Ogata Kūshū 緒方香州 en suivant la classification du Catalogue de l'ère Zhenyuan. Un travail d'investigation de ces textes a été entrepris par le Département de la Culture à partir de 1994. Il devrait se solder par un rapport détaillé. 
5. Le Hōryūji 法隆蒋. Le canon de ce monastère comporte 1262 rouleaux dont 401 de l'époque de Nara (710-781) et 625 de l'époque de Heian (781-1183). Le travail de copie du canon s'étant échelonné sur une longue période, on ne peut savoir avec certitude quels catalogues on a pris pour modèle. Pour ce qui est des copies de l'époque de Heian, on s'est, semble-t-il, fondé sur celui de l'ère Zhenyuan. Parmi elles, on compte notamment une copie du deuxième rouleau des Biographies des moines éminents des Grands Tang en quête de la Loi dans les contrées occidentales (Ta Tang Xiyu qiufa gaoseng zhuan 大唐西域求法高僧嗦) [T. 2066] de Yijing 義浮(635-713), ainsi qu'une copie des rouleaux 6, 8, 9, 11 et 12 du Catalogue de la dynastie des Grands Zhou (Da Zhou kanding zhongjing mulu 大周刊定眾經目錄) [T.2153, compilé en 695], dont on a fait une étude purement formelle portant sur les mensurations, non sur le contenu.

6. Le Natori Shingūji 名取新宮寺. 3031 rouleaux. Les copies conservées dans ce canon ont été faites aux époques Heian et Kamakura. Le choix des textes semble se fonder également sur le Catalogue de l'ère Zhenyuan. Les numéros 2349 à 2366 du catalogue de cette collection semblent être le Sütra des noms de buddha en 16 rouleaux ${ }^{1}$. Il existe également deux rouleaux : le $6^{\mathrm{e}}$ juan du Kinguanming jing (金光明經 卷第六) et le $3^{\mathrm{e}}$ juan du Xiukongzang suowen jing (虚空藏所問經 卷第三), qui sont classés parmi les textes exclus du canon par le Catalogue de l'ère Zhenyuan sans être toutefois des « apocryphes 》 (yijing 疑經) [ce sont des extraits de sūtra déjà inclus dans le canon].

7. Le Ishiyamadera 石山寺. Le canon de ce monastère comporte 4485 fascicules. Le travail de copie de ce canon s'est concentré à la fin de l'époque de Heian (milieu Xe siècle - 1183), mais il s'est poursuivi jusqu'à la fin de l'époque Momoyama (1569-1615). Les copies faites au début et au milieu de l'époque de Heian (784 - milieu X' siècle) l'ont été à partir du canon copié à l'époque de Nara, tel que celui conservé au Tōdaiji 東大寺 et au Daianji 大安寺 à Nara, ainsi que celui des monastères de Kyōto, comme le Enryakuji 延砶寺 ou le Kanjin'in de Gion 祇園感神院. Ces copies faites à la fin de l'époque de Heian suivent l'édition du canon des Song. Dans cette collection une copie du 11' rouleau du Catalogue de la dynastie des Grands Zhou est probablement identique à celles des Nanatsudera et du Myōrenji, mais diffère de celle éditée dans le Taishō.

8. Le canon ayant appartenu au sanctuaire shintō Matsunoosha 松尾社 de Kyōto conservé maintenant au monastère bouddhique Myōrenji 妙莲寺, fut copié à la fin de l'époque de Heian. Il devait comporter 4000 rouleaux environ. Considéré longtemps comme perdu, il fut retrouvé il y a deux ans, sauf 45 rouleaux conservés au monastère Hōnen-in 法然院 de Kyōto et un rouleau conservé au musée national de Kyōto.

La découverte de ce canon est due au professeur Nakao Takashi 中尾㹃, de l'Université Risshō (Tōkyō), qui l'a exhumé au Myōrenji, attenant au sanctuaire de Matsunoo, quelque 4000 rouleaux datant de la fin de l'époque de Heian ${ }^{2}$. La copie de ce canon semble avoir été entreprise à l'instigation de la puissante famille des Hata 秦氏, entre les années 1115 et 1163 environ. Mais la collection inclut cependant des textes extérieurs à cette entreprise, telles des copies du canon conservé au Jizō-in 垉藏院 et une version du Mahāprajñäpāramitā-sūtra qui est de présentation, de facture et de calligraphie différentes des autres copies ${ }^{3}$.

1. [Natori Shingūji issaikyō chōsa hōkokusho各取新宮寺一-切經 調查 報告書, éd. par Tōhoku rekishi shiryōkan 東北笔史資料館, Sendai, 1980.]

2. Nakao Takashi, “Shinhakken no Matsunoosha issaikyō ni tsuite新發現の松尾社一切經に ついて» (Nouvelle découverte du canon bouddhique du sanctuaire de Matsunoosha), Rakusei洛西 (publié par le bureau du Matsunoosha).

3. Cette copie est constituée de rouleaux de papier bleu foncé sur lequel on a collé un papier en couverture. Sur la couverture ainsi que sur les pages de garde, on a dessiné des représentations de la Terre pure et des enfers. L'axe du rouleau est décoré de nacre et de motifs dessinés, cf. Nakao Takashi, op. cit., p. 7. 
Un examen des manuscrits révèle que les copies de Matsunoosha appartiennent à la lignée du canon copié à l'époque de Nara. Les canons copiés à partir du XIe siècle ont en général été considérés comme ayant peu de valeur du fait qu'on n'y voyait que des répliques du canon des Song. Mais il en va tout autrement s'agissant de textes reproduisant le canon copié à l'époque de Nara. Toutefois un examen minutieux des manuscrits de cette collection du sanctuaire shintō s'impose : outre qu'ils comportent des erreurs de copistes, les manuscrits sont souvent endommagés par les insectes ou l'humidité. Leur utilisation s'avère donc délicate pour établir des textes critiques, d'autant qu'il n'est pas aisé d'en prendre des photographies, pour des raisons notamment financières. Il faudra sans doute attendre au moins trois années avant qu'on en dresse un catalogue détaillé.

9. Le Daitokuji 大德寺, grand monastère Zen de Kyōto. Sa collection, qui devait comporter environ 6000 rouleaux, n'a pas été compilée ni copiée en une fois. C'est tout au long des époques de Muromachi (1392-1569) et d'Edo (1615-1867) qu'on a, à plusieurs reprises, rassemblé les textes qui la constituent. Sa teneur est, pour ce motif, souvent composite. On y trouve plusieurs centaines de textes appartenant au canon copié à l'époque de Nara, ainsi que des spécimens des collections du Bonshakuji 梵程寺 et du Kōzanji 高山寺 qui sont représentatives des copies de l'époque de Heian. Les lacunes sont comblées avec l'édition dite Obaku 黃檗版 (1669-1681). On ne semble pas avoir cherché à constituer un canon complet, mais seulement à rassembler des copies anciennes qui commençaient à passer au second plan devant la vogue, à la fin de l'époque de Heian et au cours de celle de Kamakura (1183-1331), des éditions imprimées.

\section{La découverte de manuscrits considérés comme perdus aux Nanatsudera}

Les Nanatsudera de Tōenzan 稻園山七寺, sont un monastère Shingon de la branche Chizan 真言宗智山派. Situé dans la ville de Nagoya, préfecture d'Aichi, le monastère se trouve dans un environnement privilégié, entouré qu'il est par le sanctuaire shintō d'Atsuta 熱田神宫 et le monastère bouddhique d'Osu Kannon 大須觀音. La légende en attribue la fondation au moine Gyōki 行基 (668-749), à l'époque de Nara. On sait seulement de façon certaine qu'à la fin de l'époque de Heian, le gouverneur surnuméraire de la province d'Owari 尾㖘 (actuelle préfecture d'Aichi), Onakatomi no Yasunaga 大中臣安長, a apporté sa protection financière et son soutien politique pour la copie du canon bouddhique. Deux moines se sont chargés de l'entreprise, Eigei 柴芸 et Eishun 笨俊. Ils avaient pratiquement achevé la copie de 300 rouleaux entre 1175 et 1178. Par la suite, ils ont conçu le projet de copier des sūtra, des traités et des commentaires non inclus dans le canon. Mais leur travail semble avoir été interrompu en 1180.

Le canon des Nanatsudera a été désigné «Trésor national » en 1900, puis, dans les années qui ont suivi la fin de la seconde guerre mondiale, il est devenu «Bien culturel important ». On a alors organisé des missions d'enquête qui se sont soldées par la publication en 1968 du Catalogue du canon bouddhique des Nanatsudera, Matériaux historiques de la province d'Owari (尾張史料.七寺一切經目铮). Ce catalogue a été distribué à des institutions universitaires ainsi qu'à des chercheurs, mais curieusement, personne ne semble y avoir prêté attention. La raison en est que, jusqu'à présent, on a considéré que les copies du canon bouddhique effectuées à la fin de l'époque de Heian reproduisaient le canon de l'édition des Song. Ce préjugé tenace est pourtant sans fondement scientifique. Certes, l'édition impériale faite durant l'ère Kaibao des Song septentrionaux (北宋敕顾開貲藏) (968-976), apportée du continent par le moine Chōnen (938-1016) en 986, a constitué un texte de base important qui était fort prisé. Cependant, les milieux qui y avaient accès étaient fort limités.

Par ailleurs, de nombreux textes rares non inclus dans les catalogues ont été imprimés dans de grandes collections japonaises, comme le Canon bouddhique compilé sous l'ère Taishō (Taishō shinshū daizōkyō大正新䚘大藏經), le Corpus bouddhique du Grand Japon 
(Dainihon bukkyō zensho大日本佛教全書), le Canon bouddhique du Japon (Nihon daizōkyō 日本大藏經), la Suite au Canon bouddhique (Zokuzōkyō 續藏經), les CEuvres completes de la secte Jōdo (Jōdoshū zensho 浮土宗全茟), les CEuvres complètes de la secte Shingon (Shingonshū zensho 真言宗全茟) ou les Cuvres completes de la secte Tendai (Tendaishü zensho 天台宗全書). Mais en raison du fait qu'ils se présentaient sous une forme déjà imprimée, trop accessible peut-être, on n'y a pas accordé l'intérêt qu'ils méritaient. En outre, les spécialistes du bouddhisme au Japon se sont surtout penchés sur l'Inde et le Tibet et n'ont guère pris en considération la Chine et le Japon, si ce n'est dans une optique sectaire. Les meilleurs chercheurs travaillant sur les manuscrits chinois se sont malheureusement concentrés presque exclusivement sur les manuscrits de Dunhuang et non sur ceux conservés au Japon, plus ou moins convaincus qu'ils étaient qu'aucun manuscrit nouveau touchant le bouddhisme chinois ne pouvait y être retrouvé à l'heure actuelle.

Or, pendant une période prolongée, la Corée et le Japon se sont trouvés sous l'influence culturelle de la Chine où fleurissait le bouddhisme. Le peu que laisse entrevoir la richesse des manuscrits de Dunhuang fait imaginer qu'il devrait en être de même dans les autres régions périphériques de la Chine qu'ont été la péninsule Coréenne et le Japon. Toutefois, la question était de savoir si des documents d'importance y subsistaient ou non. L'opinion prévalait que presque plus aucun manuscrit ne pouvait y être retrouvé.

Au Japon cependant, on a conservé plusieurs milliers de textes copiés à l'époque de Nara et plusieurs dizaines de milliers pour l'époque de Heian. Ceux de l'époque de Nara reproduisent fidèlement les versions qui avaient cours sous les Tang. Ce sont eux qu'on aurait dû utiliser en priorité pour établir les éditions critiques reproduites dans les grandes collections comme le Canon imprimé sous l'ère Taishō. En effet, ce dernier s'est fondé sur les versions coréennes qui datent du XII ${ }^{e}$ siècle, en mettant en parallèle les variantes que présentent les éditions des Song, des Yuan et des Ming. Elles sont toutes éloignées dans le temps des éditions des Tang, qui marquent l'apogée du bouddhisme. Le canon copié au Japon à l'époque de Nara a en fait une plus grande valeur que celui des Song, car il est plus fidèle aux originaux que lui.

Je prendrai pour exemple la Biographie d'Aśvaghoṣa traduite par Kumārajīva. Une copie datée de la fin de l'époque de Heian est conservée aux Nanatsudera et au Kōshōji. La version du Taishō (éditions coréenne, des Song, des Yuan et des Ming) [T. 2046] en diffère sensiblement. À l'époque où s'est constitué le Catalogue de l'ère Kaiyuan établi par Zhisheng (669-740) en 730, la version de cette biographie, largement recopiée au Japon à l'époque de Heian, devait présenter une teneur identique à une version provenant des grottes de Dunhuang, si une telle version s'avérait y être conservée.

Ainsi, les canons bouddhiques recopiés à l'époque de Heian à partir de la copie de l'époque de Nara, reproduisent de ce fait fidèlement des versions qui avaient cours à l'époque des Tang. On pourrait par voie de conséquence déduire, à partir de leur examen minutieux et systématique, ce que devait être le canon de l'époque des Tang.

La plus grande partie des textes conservés aux Nanatsudera proviennent d'ouvrages qu'on a recopiés dans des monastères de la province d'Owari. Cependant l'entreprise ne s'est pas limitée à cette seule province. Elle a nécessité un grand déploiement de moyens et d'énergie qui n'a été rendu possible que grâce à l'influence de la famille des Onakatomi, et en particulier de Yasunaga, sur les sanctuaires shintō et les monastères bouddhiques. Celui-ci a cherché à se procurer des textes jusque dans la région de Kyōto.

Il est important de noter qu'on a suivi la structure donnée par le Catalogue de l'ère Zhenyuan. Ce catalogue, tel qu'il est édité dans le Taishō, ne correspond pas à la version primitive ainsi que l'a montré Tsukamoto Zenryū 塚本善隆 ${ }^{1}$. Le canon des Nanatsudera s'est

1. [Tsukamoto Zenryū, « Nihon ni izonsuru gempon Jōgen shintei shakkyō mokuroku日本に遺存する 原本貞元新定䆁教目錄 》, Shoshigaku ronshū 曺誌學諭集, Kyōto, 1957, p. 423-435.] 
fondé, outre sur des manuscrits d'Owari, sur ceux conservés dans trois temples de Kyōto qu'on a utilisés afin de faire un travail de collationnement : ce sont ceux mentionnés plus haut par ordre d'importance (1) du Hōshōji, (2) de Fushimi et (3) du Bonshakuji (焚䆁寺). Ensuite, le Catalogue de l'ère Zhenyuan, qui se trouve dans ces trois monastères, mentionne une « liste d'ouvrages non inclus dans le canon ", dont plus de la moitié était conservée au Hōshōji et au Bonshakuji. On a donc cru devoir les recopier scrupuleusement et systématiquement.

En conclusion, on peut avancer que : 1. le canon des Nanatsudera a pris pour modèle celui du Hōshōji ; 2. les moines qui ont copié ce canon ne comprenaient pas le sens exact du terme « ouvrages non inclus dans le canon». Le Hōshōji est un grand monastère impérial représentatif du début de l'époque de Heian, et le Bonshakuji est un grand centre d'études scolastiques représentatif de la fin de la même époque. Il est naturel que tous deux aient hérité de la tradition des Sept monastères de Nara, que sont le Tōdaiji 東大寺, le Kōfukuji 興福寺, le Gangōji 元興寺, le Daianji 大安寺, le Yakushiji 樂師寺, le Saidaiji 西大寺 et le Hōryūji 法隆寺.

La « liste d'ouvrages non inclus dans le canon 》 (不入藏目錄) recense ceux que Zhisheng a écartés du canon pour plusieurs raisons :

1. Les textes déjà intégrés dans la Somme du Grand sūtra aux amas de joyaux [Mahäratnaküta-sūtra ou Da baoji jing 大留經] [T. 310].

2. Les versions d'un même texte, mais comportant un nombre de rouleaux différent en raison d'un autre découpage.

3. Des textes déjà catalogués sous un autre titre.

4. Des textes figurant deux fois dans le Catalogue de la dynastie des Zhou, constitué en 695 sous l'impératrice Wu Zetian 武則天 (624-705).

5. Des extraits de sūtra copiés comme sütra complets.

6. Des récits tirés du vinaya des Mūlasarvāstivādin et donnés comme textes complets.

7. Des ouvrages répertoriés dans le Catalogue des Zhou, mais qui ont été perdus.

8. Des textes douteux et apocryphes.

Zhisheng dénombre en tout 118 titres pour 247 rouleaux [T. 2157 (30), LV, 1046b1048a].

Ces textes étaient recopiés et étudiés à l'époque de Nara en dépit de leur caractère « extracanonique ». Or il se trouve que pour des raisons historiques les Nanatsudera, qui ont hérité de cette tradition de Nara, sont le monastère qui en a conservé le plus grand nombre, soit 13 titres et 105 rouleaux, dont certains sont des unica.

\section{Révevaluation des copies du canon bouddhique faites à l'époque ancienne au Japon}

J'espère avoir donné une idée de l'importance des canons bouddhiques recopiés au Japon à époque ancienne. Pour ne pas prêter à méprise, je voudrais rajouter que, loin de faire peu de cas des éditions actuellement imprimées du canon bouddhique (Taishō) ainsi que des manuscrits de Dunhuang, c'est tout-à-fait conscient de leur importance que j'ai poursuivi mes recherches et en ai présenté les résultats. Il me semble pourtant que le Taishō et les manuscrits de Dunhuang doivent être étudiés en même temps que le canon d'époque des Tang, aujourd'hui conservé dans celui de Nara et dans ceux de l'époque de Heian qui le prolongent. Les canons copiés au Japon ont eu tendance à être gardés jalousement secrets en raison de leur haute valeur artistique et esthétique ; on n'a jusqu'à présent relativement peu eu l'occasion de se pencher sur eux en tant que documents, dans une optique philologique.

Aujourd'hui, quiconque le veut est en mesure de consulter des éditions en facsimilé de l'édition coréenne, de même que celle de Qisha des Song (1238) ou de Jiaxing des Ming (1522-1566). Les manuscrits de Dunhuang sont reproduits en microfilms, édités en facsimilé, et l'on commence à en publier des éditions photographiques très accessibles et lisibles.

Mais qu'en est-il actuellement des copies du canon au Japon ? Pas l'ombre d'un projet pour les prendre en microfilm ou en photographie sur une grande échelle. À tout le moins, un 
organisme officiel devrait se donner pour tâche d'entreprendre ce travail pour le canon de Nara. Au Japon, on accorde un prix extrême aux biens culturels du pays. Mais on ne fait que les garder comme des détenus dans une prison, et on adopte une politique de protection du patrimoine telle qu'on ne les rend que trop rarement publics, comme si l'on craignait de les exposer en plein jour.

Traduit et adapté par Frédéric GIRARD et KUO Li-ying 